19. Fortbildungskongress, Davos 2003

\section{Herzlich willkommen zum Drei-Länder-Treffen!}

$Z$ um fünften Mal findet im September 2003 das Drei-Länder-Treffen der Deutschen, Österreichischen und Schweizerischen Gesellschaften für Allergologie und klinische Immunologie statt. Die Idee zu einem derartigen Treffen geht auf die 80er Jahre zurück als der damalige Präsident der Deutschen Gesellschaft für Allergologie und klinische Immunologie (DGAI), Prof. Dr. Wolfgang Schmutzler, den Wunsch verschiedener junger Kollegen aufgriff und ein solches Treffen anregte. Erstmals kam es 1993 dann in Gaschurn, Vorarlberg, zur Realisierung. Einige Jahre später traf man sich anlässlich des Fortbildungskongresses „Fortschritte der Allergologie, Immunologie und Dermatologie" in Davos, wobei die drei Gesellschaften jeweils vier Redner und Themen vorschlagen durften.

In der jüngeren Vergangenheit wurde die Planung unterschiedlich gehandhabt. Während manchmal eine Gesellschaft ein Symposium für sich thematisch gestalten wollte, war es in der Mehrzahl der Fälle doch sinnvoller, den Themen folgend einfach länderübergreifend attraktive Symposien zu gestalten. So ist man auch in diesem Jahr verfahren. Während die Schweizer

\section{Wissenschaftliches Programm}

\begin{tabular}{ll}
\hline Thema & Seite \\
Vorträge & \\
Festvortrag & 368 \\
Atemwegsallergie und Asthma & 368 \\
Allergie und Haut & 369 \\
$\begin{array}{l}\text { Immunmodulation in der } \\
\text { Psoriasistherapie }\end{array}$ \\
$\begin{array}{l}\text { Grundlagen der Allergie } \\
\text { Klinische Allergologie }\end{array}$ \\
$\begin{array}{l}\text { Sublinguale Hyposensibilisierung } \\
\text { Anaphylaxie }\end{array}$ & 370 \\
Neue Therapien & 372 \\
Poster & 377 \\
\hline
\end{tabular}

Kollegen das Symposium „Anaphylaxie“ am Samstagvormittag ausschließlich gestalten, bringen sich die österreichischen Allergologen sowohl bei den „Grundlagen der Allergie“ als auch bei den klinischen Symposien ein. Ein Novum ist die Teilnahme von Kolleginnen und Kollegen aus dem benachbarten Südtirol, die sich in diesem Jahr erstmals auch aktiv mit Beiträgen an der Programmgestaltung beteiligen.

Die Idee des Drei-Länder-Treffens resultiert nicht nur aus dem Wunsch zur Pflege gut nachbarlicher Beziehungen zwischen den Allergiegesellschaften und den praktizierenden Kolleginnen und Kollegen. Sie hat ihren tieferen Grund auch in der gemeinsamen Philosophie der fächerübergreifenden Allergologie, die auf dem Boden von organbezogenen Disziplinen in einer durchaus qualitativen wertvollen Weiterbildung dann fächerübergreifend betrieben wird. Diese mitteleuropäische Tradition ist in unseren drei Ländern besonders ausgeprägt und muss sich im Rahmen der Harmonisierungsbestrebungen in Europa gegen unterschiedliche Modelle behaupten, die aus den romanischen Ländern oder dem angelsächsischen Raum kommen.

Das bunte und reichhaltige Programm ist auf die praktizierenden Kollegen ausgerichtet und trägt dem interdisziplinären Charakter der Allergologie Rechnung. Neben grundlagenbezogener Forschung kommen klinische Themen und Fragen der Therapie zur Diskussion. Am Nachmittag erhalten die Teilnehmer in kleinen Gruppen Gelegenheit, zu praktischen Übungen auf verschiedenen Teilgebieten. Jeder Tag beginnt mit einer „Dia-Klinik“ in Form von illustrativen und lehrreichen Fallvorstellungen. Auch gesundheits- und standespolitische Themen werden zu Wort kommen. Ein Höhepunkt des Kongresses wird der Eröffnungsabend mit der Verleihung des Kanert-Preises für Allergieforschung und dem Festvortrag von Prof. Happle über

\section{Kongressdaten auf einen Blick}

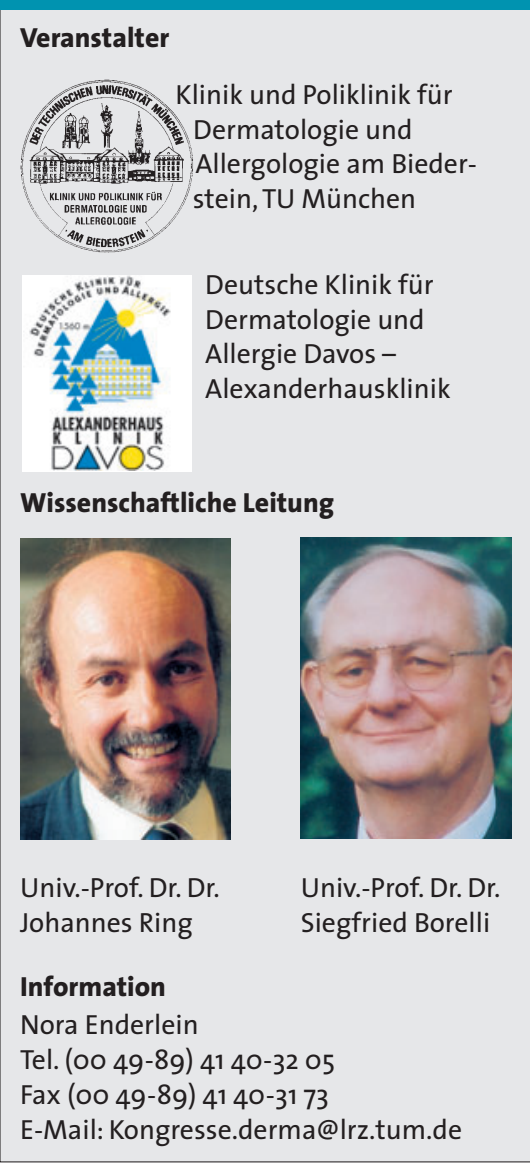

„Haut-Mosaike“ sein. Eine Posterausstellung gibt jungen Wissenschaftlern Gelegenheit, aktiv originelle Forschungsergebnisse vorzustellen. Selbstverständlich tragen auch die in Davos ansässigen Institutionen, insbesondere die Alexanderhausklinik, aber auch die Hochgebirgsklinik Wolfgang und natürlich das Schweizerische Institut für Allergie- und Asthmaforschung (SIAF) ganz wesentlich zur Programmgestaltung bei.

Ich wünsche allen Teilnehmern interessante Tage, gute Diskussionen, freundschaftliche Begegnungen und den Allergiegesellschaften der drei Nachbarländer eine aktive weitere Entwicklung!

Ihr

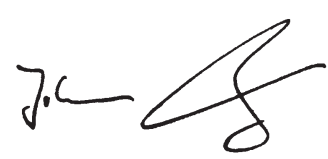

Univ.-Prof. Dr. med. Dr. phil. J. Ring 